\title{
Production and characterization of monoclonal antibodies against amphiphysins
}

\author{
YuLian Jin', Kyung-Yong Kim', \\ Nak-Kyun Soung', Eun-Young Shin', \\ Eung-Gook Kim ${ }^{1}$ and Seung Ryul Kim ${ }^{1,2}$ \\ ${ }^{1}$ Department of Biochemistry, College of Medicine, Medical Research \\ Institute, Chungbuk National University, Cheongju, Korea \\ ${ }^{2}$ Corresponding author: Tel, +82-43-261-2860; \\ Fax, +82-43-272-1603; E-mail, srkim@med.chungbuk.ac.kr
}

Accepted 30 May, 2001

Abbreviations: Amph1; amphiphysin I, Amph2; amphiphysin II, SDS-PAGE; sodium dodecylsulfate-polyacrylamide gel electrophoresis, MAb; monoclonal antibody, GST; glutathion S-transferase

\begin{abstract}
Amphiphysin I and II, proteins enriched in nerve terminals, form heterodimers and interact with dynamin and synaptojanin through their Src homology 3 (SH3) domain. In order to study the expression profile of Amphs in cells and tissues and the interaction state with other cellular molecules, we have prepared specific monoclonal antibodies (mAbs) designed to bait $\mathrm{N}$-terminus, middle part, and $\mathrm{C}$-terminus domains of Amph I, respectively by immunizing with the expressed smaller domain molecules using the GST gene fusion system. The expression of Amphs was found to be most abundant in PC12 cells, followed by B103 cells and vascular smooth muscle cells. Western blot analysis showed a relatively high level expression of Amphs that were found in both mouse and rat brain. There appeared to be some species difference in the expression pattern, i.e. Amphs are present more in the testis than in the lungs in rats, however, they are reversed in mice. Characterization of the mAbs revealed that clone 14-23 precipitated Amph I and II, whereas clone 8-2 could only precipitate Amph I. In addition, clathrin and dynamin in a complex with Amph were captured in the precipitate formed by mAbs and identified by the Western blot analysis. Cellular distribution of Amph was visualized with confocal immunofluorescence microscopy performed using the labeled-mAbs. Taken together, these results demonstrated that mAbs provided an excellent measure for studying Amphs' expression profile and their interacting proteins.
\end{abstract}

Keywords: amphiphysin, monoclonal antibody

\section{Introduction}

Amphiphysin I (Amph1) and amphiphysin II (Amph2) are nerve terminal-enriched proteins thought to function in synaptic vesicle endocytosis, in part through Src homology 3 (SH3) domain-mediated interactions with dynamin and synaptojanin (Wigge et al., 1997). In 1992, Amph1 was discovered (Lichte et al., 1992), and recently Amph2 has been identified as an isoform of Amph1 (Ramjaun and McPherson, 1998). Amph2 has various splice variants, and the $95 \mathrm{kD}$ isoform is predominant in the brain. Amph1 and Amph2 form a heterodimer in the brain, which suggests that the two isoforms may function as a complex (Wigge et al., 1997; Wigge and McMahon, 1998). Dephosphorylation of Amph1 requires extracellular $\mathrm{Ca}^{2+}$ (Bauerfeind et al., 1997) and dephosphorylation of rat brain extract was shown to promote the assembly of dynamin1, and synaptojanin1 into complexes that also included clathrin and AP-2 (Slepnev et al., 1998). Thus, phosphorylation regulates the association and dissociation cycle of the clathrin-based endocytic machinery, and calcium-dependent dephosphorylation of endocytic proteins could prepare nerve terminals for a burst of endocytosis. Recently, it has been reported that calcineurin-binding protein cain stably associated with the $\mathrm{SH} 3$ domain of Amph1 without affecting the interaction of amphiphysin with other endocytic proteins, and negatively regulated synaptic vesicle endocytosis by inhibiting the activity of calcineurin (Peters et al., 2000). Amph2 has been suggested to have an additional role in addition to endocytosis because it has the ability to interact with the Ras-GTP exchange factor SOS and may function as a link between endocytosis and receptor-mediated signaling (Leprince et al., 1997). We have previously shown that amphiphysins inhibit the activity of phospholipase D (PLD) by interaction with PLD (Lee et al., 2000). Amphiphysins may play an important physiological role both in mitosis and in differentiated cells because it binds the cdk5 regulatory subunit p35 and is phosphorylated by cdk5 and cdc2 (Floyd et al., 2001).

It became important to investigate the change of binding proteins with Amphs because the physiological roles of Amphs are affected according to the change of binding proteins with Amphs. Thus, to study the change of binding proteins with Amphs, Amph1 was purified using the GST fusion system and the monoclonal anti- 
bodies (mAbs) against Amph1 were produced and characterized. At least, the antibodies prepared recognized three different epitopes, $\mathrm{N}$-terminal, C-terminal, and the middle part of Amph1. In addition, we found that clone1 precipitated both of the Amphs and that clone 2 precipitated only Amph1.

\section{Materials and Methods}

\section{Materials}

The following reagents were obtained from Amersham (Buckinghamshire, UK): ECL kit, PVDF membrane, glycine, Tris, SDS, acrylamide. Brilliant blue G 250 and pristane (2,6,10,14-tetramethyl pentadecane), PEG, HRP anti-mouse IgG, FITC and anti-amphiphysinll MAb were obtained from Sigma (Louis, MO, USA). DMEM medium, FBS, and IPTG were obtained from GibcoBRL (Burlington, Ontario, Canada). Cell culture dish, plate and ELISA plate were obtained from Nunc (Naperville, IL, USA). Glutathione sepharose 4B was obtained from Pharmacia. Complete and incomplete Freunds adjuvants were obtained from GibcoBRL. BCIP/NBT was obtained from KPL (Maryland, USA).

\section{Purification of GST-amphiphysin fusion proteins}

Truncated cDNAs encoding the $\mathrm{NH}_{2}$-terminal 373 residues (Amph1N) or $\mathrm{COOH}$-terminal 404 residues (Amph1C) of Amph1 from human were separately ligated into the $5^{\prime}$ BamHI and 3' EcoRI sites of the pGEX4T vector (Amersham Pharmacia Biotech) by standard techniques of subcloning and the polymerase chain reaction to produce expression vectors (pGEX-Amph1N and pGEX-Amph1C) encoding the respective GST fusion proteins. Escherichia coli BL21 cells were transformed with the various expression vectors encoding the GST fusion proteins and grown at $37^{\circ} \mathrm{C}$ until the absorbance at $600 \mathrm{~nm}$ of the culture reached 0.5-0.6. Expression of GST-Amph proteins was then induced by incubation of the cells for $3 \mathrm{~h}$ in the presence of $100 \mu \mathrm{M}$ isopropyl- $\beta$-D-thiogalactopyranoside (IPTG). Cells were collected, washed with phosphate-buffered saline (PBS), sonicated in PBS containing 1\% (v/v) Triton X-100, 2 mM EDTA, 1 mM phenylmethylsulfonyl fluoride, and centrifuged at $25,000 \mathrm{~g}$ for $30 \mathrm{~min}$. GST fusion proteins were purified from the resulting supernatant with the use of glutathione-Sepharose 4B (Amersham Pharmacia Biotech).

\section{Production of monoclonal antibody against amphi- physins}

GST-Amphs, purified as described above, were injected into BALB/c mice. Hybridomas were prepared by fusing spleen cells with cells of myeloma line SP2/0-Ag14 using previously described procedures (Lee et al., 1996). Enzyme linked immunosorbent assay (ELISA) were performed to insure that each monoclonal antibody selected reacts exclusively with Amph. The prepared antibodies recognized at least three different epitopes of Amph1.

\section{Enzyme linked immunosorbent assay}

Ninety-six well microtiter plates (Nunc-Immuno Plate, MaxiSorp ${ }^{\mathrm{TM}}$ ) were coated with the Amph1C or Amph1N (protein concentration; $5 \mu \mathrm{g} / \mathrm{ml}$ ) by incubating plates overnight at room temperature (under shaking). Plates were then washed twice with PBS containing $0.05 \%$ Tween 20 (PBS-T, $200 \mu \mathrm{l} /$ well), incubated with $2 \%$ BSA in PBS $(100 \mu \mathrm{l} /$ well) for $1 \mathrm{~h}$ at room temperature, and washed again twice with PBS-T $(200 \mu \mathrm{l} /$ well). In parallel, supernatants of selected clones were incubated for $1 \mathrm{~h}$ at $37^{\circ} \mathrm{C}$. Subsequently, anti-mouse Ig antibody (Sigma, $1: 3,000)$ linked to horseradish peroxidase was incubated for another $1 \mathrm{~h}$ at room temperature. After this incubation step, plates were washed with PBS-T (four times, $200 \mu \mathrm{l} /$ well). Enzymatic reactions were carried out at room temperature by adding $\mathrm{H}_{2} \mathrm{O}_{2}$ and o-phenylenediamine $(2.5 \mathrm{mM}$ each in $0.1 \mathrm{M}$ phosphate-citrate buffer, $\mathrm{pH} 5.0)(50 \mu \mathrm{l}$ of each reagent/well). Reactions were stopped after $30 \mathrm{~min}$ by the addition of $50 \mu \mathrm{l}$ of $1 \mathrm{M}$ $\mathrm{H}_{2} \mathrm{SO}_{4}$ containing $0.05 \mathrm{M} \mathrm{Na}_{2} \mathrm{SO}_{3}$. Color development was measured at $405 \mathrm{~nm}$. To exclude the monoclonal antibodies that recognize GST, control ELISA was performed in parallel with GST.

\section{Immunoprecipitation and Western blotting}

Cells and tissues were homogenized in 5 volumes of $10 \mathrm{mM}$ Hepes ( $\mathrm{pH} 7.4$ ) containing $1 \mathrm{mM}$ EDTA in the presence of a protease inhibitor cocktail (PIC; aprotinin, antipain, leupeptin, and pepstatin each at $3 \mu \mathrm{g} / \mathrm{ml})$. The lysate was centrifuged at $20,000 g$ for $1 \mathrm{~h}$. The supernatants were precleared with protein A-gel and then subjected to immunoprecipitation with a monoclonal antibody to amphiphysins and protein A-gel. Immune complexes were collected and washed by a brief centrifugation, separated by SDS-PAGE on an $8 \%$ gel. The proteins in the gel were then transferred onto nitrocellulose membrane. Western blotting was performed with monoclonal antibodies to amphiphysins using an ECL kit. Protein quantification of samples was performed using the method of Bradford (1976).

\section{Cell culture}

The cell lines were cultured in Dulbecco's modified Eagle's medium supplemented with $10 \%(\mathrm{v} / \mathrm{v})$ fetal bovine serum, 100 units of penicillin $/ \mathrm{ml}$, and $100 \mu \mathrm{g}$ of streptomycin $/ \mathrm{ml}$ at $37^{\circ} \mathrm{C}$ in a humidified, $\mathrm{CO}_{2}$-controlled (5\%) incubator. Cells were maintained to keep subconfluency (about $80-90 \%$ ) and serially passaged. B103 and PC12 cell line are neuronal cell lines, F9 cells are the teratocarcinoma cells of mouse, ECV304 is an endothelial cells, and vascular smooth muscle cells (VSMC) 
was prepared from the aorta of rat.

\section{Confocal immunofluorescence microscopy}

Vascular smooth muscle cells (VSMC) were fixed and permeated in $2 \%$ paraformaldehyde/0.1\% Triton X-100 for $15 \mathrm{~min}$ at room temperature. Confocal immunofluorescence microscopy was performed for VSMC by incubating cells with a monoclonal antibody against amphiphysins for $90 \mathrm{~min}$ at $37^{\circ} \mathrm{C}$. Primary antibody was detected using FITC-conjugated secondary antibody (molecular probe, USA) for $30 \mathrm{~min}$ at $37^{\circ} \mathrm{C}$. Washes were performed with $\mathrm{PBS} / 0.1 \%$ Triton $\mathrm{X}-100$ after both primary and secondary antibody incubation. Cells were mounted in Anti-fade and visualized using a confocal microscope (Bio Rad, MRC 1024, USA).

\section{Results}

\section{Purification of GST-Amph1C and GST-Amph1N}

The expressed GST fusion proteins were purified from the supernatant with the use of glutathione-Sepharose 4B (Figure 1). Even though the purified fraction of GST-

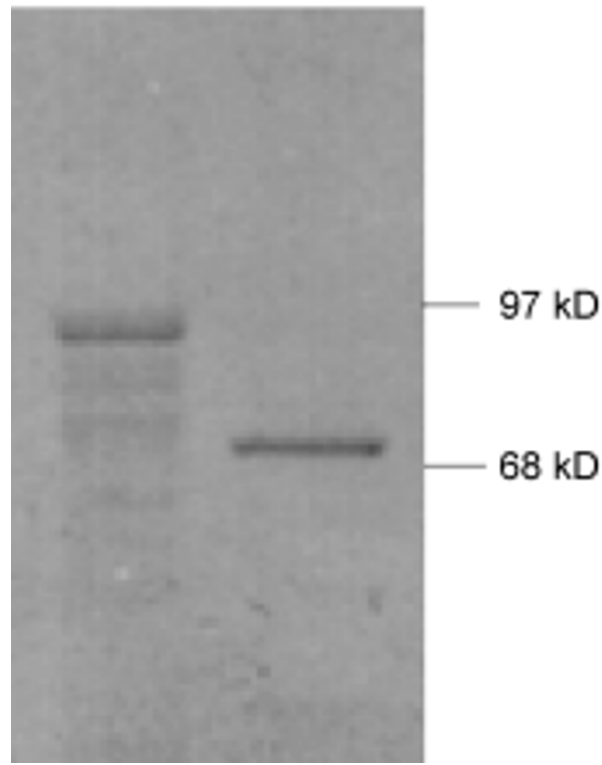

Figure 1. Purified GST-Amph1C and GST-Amph1N. Escherichia coli BL21 cells were transformed with the pGEX4T vectors encoding the GST fusion proteins and grown at $37^{\circ} \mathrm{C}$ until the absorbance at $600 \mathrm{~nm}$ of the culture reached 0.5-0.6. Expression of GST-Amph proteins was then induced by incubation of the cells for $3 \mathrm{~h}$ in the presence of $100 \mu \mathrm{M}$ isopropyl- $\beta$-Dthiogalactopyranoside (IPTG). Cells were collected, washed with PBS, sonicated in PBS containing 1\% (v/v) Triton X-100, 2 mM EDTA, 1 mM phenylmethylsulfonyl fluoride, and centrifuged at $25,000 \mathrm{~g}$ for $30 \mathrm{~min}$. GST fusion proteins were purified from the resulting supernatant with the use of glutathione-Sepharose 4B (Amersham Pharmacia Biotech). Purified samples were separated on an 8\% SDS-PAGE gel. Lane 1, purified GSTAmph1C; lane 2, purifed GST-Amph1N.
Amph1N revealed a single band, the purified fraction of GST-Amph1C was consisted of several fragments. The purified GST-fusion proteins were used as antigen to produce monoclonal antibody against Amphs.

\section{ELISA with selected clones}

To characterize selected clones for its specificity toward three different epitopes, ELISA was performed. Clones 14-23, 20-6, and 23-6 recognized only Amph1C. Clone 4-2 reacted only with Amph1N, but clones 1-1 and 8-2 bound both Amph1N and Amph1C (Figure 2). Clones 11 and 8-2 seemed to recognize the middle part of Amph1 (Amph1M).

\section{Western blot analysis with anti-amphiphysins}

The selected antibodies were further evaluated by Western blot analysis. Even though some clones did not show binding, clones 4, 8, and 14 showed strong binding in Western blot analysis. Our system could recognize about 100pg of Amph1 (Figure 3A).

To profile Amphs expression in various tissues, western blot analysis was performed on the tissue extracts of selected tissues and gland in both rat and mice i.e. brain, lung, testis, and adrenal gland. The result showed that Amphs are most abundantly expressed in brain tissues. Interestingly, the expression of Amphs was positive in the testis of rats but not in mice (Figure 3B). Cellular location of Amphs, examined by the subfractionation of cellular component showed some variation between the tissues; Amph1 is present in the cytosolic fraction of the rat lung, whereas it is present in both the cytosolic and the membrane fractions of the brain (Figure $3 \mathrm{C}$ ).

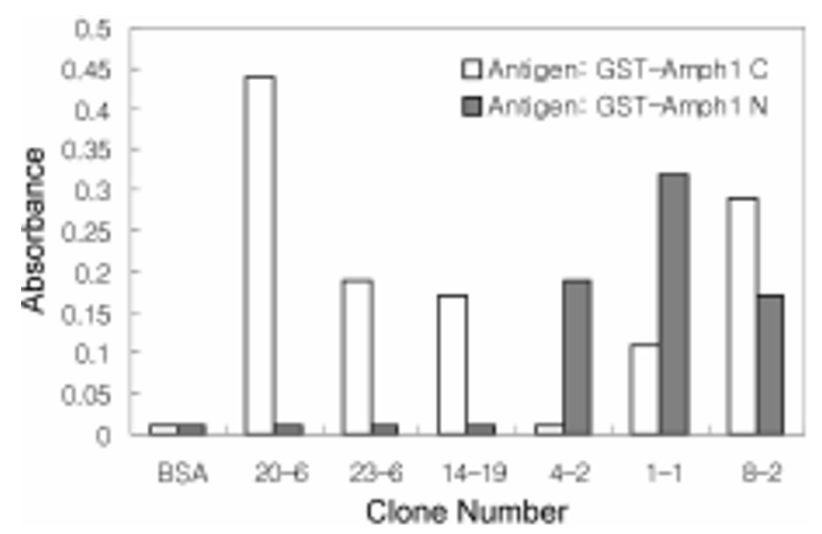

Figure 2. The result of ELISA with selected clones. Purified GST fusion proteins (GST-Amph1C and GST-Amph1N) were coated on a 96-well plate. The plate was then washed twice with PBS containing $0.05 \%$ Tween 20 incubated with $2 \%$ BSA in PBS for $1 \mathrm{~h}$ at room temperature, and washed again twice with PBS-T. In parallel, supernatants of selected clones were incubated for $1 \mathrm{~h}$ at $37^{\circ} \mathrm{C}$. Subsequently, anti-mouse Ig antibody linked to horseradish peroxidase was incubated for another $1 \mathrm{~h}$ at room temperature. After this incubation step, plates were washed with PBS-T (four times, 200 $\mu l / w e l l)$. The color was developed and read at $405 \mathrm{~nm}$. 
A

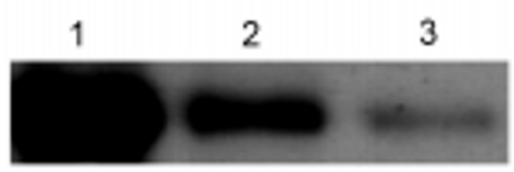

$\mathrm{C}$

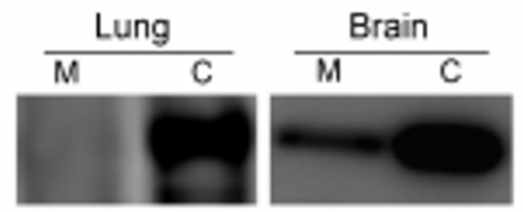

$\mathrm{E}$

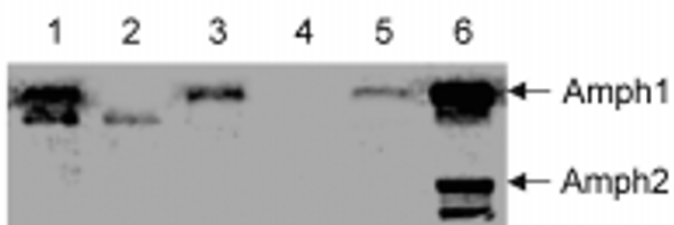

B

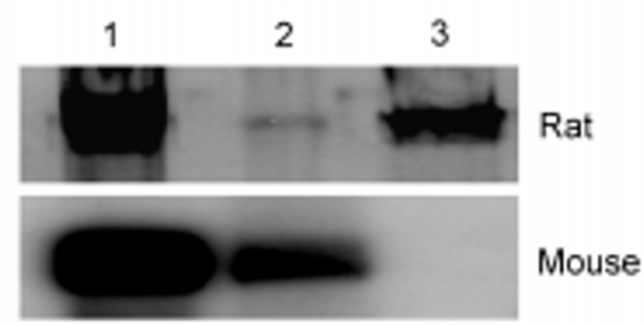

D
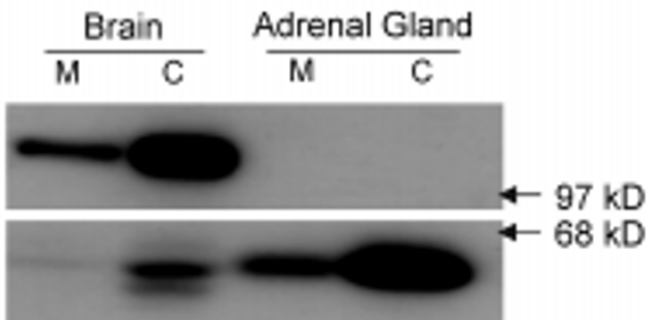

Figure 3. Western blot analysis with supernatants of selected clones. A, Purifed GST-Amph1C was separated on an $8 \%$ SDS-PAGE gel after loading different amounts of proteins. Western blot analysis was performed with supernatants of selected clones. Lane 1, 2, 3, $50.0 \mathrm{ng}, 5.0 \mathrm{ng}, 0.5 \mathrm{ng}$ each of purified GSTAmph1C. B, Homogenates of several tissues were separated on an 8\% SDS-PAGE and Western blot analysis was performed. upper panel, tissues of rat; lower panel, tissues of mouse. Lane 1, brain; lane 2, lung; lane 3, testis. C and D, Homogenates of lung, brain, and adrenal gland were separated to membrane and cytosolic fraction. Western blot analysis was performed. M, membrane fraction; C, cytosolic fraction. E, Several cell lines were harvested, solublized. The cell lysates were quantified by Bradford method and the same amounts of proteins were loaded. The cell lysates were separated on an $8 \%$ SDS-PAGE and Western blot analysis was performed. Lane 1, B103; lane 2, NIH3T3; lane 3, VSMC; lane 4, ECV304; lane 5, F9; lane 6, PC12.

Examination of several cell lines showed the relative level of Amphs expression and they are much higher in PC12 cells, followed by B103 and VSMC, but much lower in F9 cells (Figure 3D). Since PC12 cell lines are derived from chromaffin cells of adrenal glands and from transplantable rat pheochromocytoma, an examination of rat adrenal gland showed the presence of only a small level not matching those of PC12 cell lines (Figure 3E).

\section{Immunoprecipitation analysis with anti-amphiphy- sins}

The antibodies were further evaluated by immunoprecipitation analysis. Clone 8-2 precipitated only Amph1, but clone 14-23 precipitated several proteins (Figure $4 \mathrm{~A})$. The p95 band precipitated by clone 14-23 crossreacted with a commercial anti-Amph2 (Figure 4B).

To evaluate whether mAbs (Amph) recognized epitope site(s) of Amph related with the interaction with other ligands such as dynamin and clathrin, the Amph complex with either dynamin or clathrin was precipitated with mAbs (Amph) clone 14-23 and analyzed by Western blotting with anti-dynamin and anti-clathrin. Two bands of dynamin and clathrin are shown (Figure 4C). The results indicated that clone 14-23 did not interfere with the binding of dynamin and clathrin with Amphs and epitopes are structurally distant and distinct from the ligand binding sites.

\section{Confocal Immunofluorescence microscopy with anti- amphiphysins}

To confirm the earlier cellular distribution of Amphs by immunoblot analysis of cellular sub-fractions, direct identification of cellular location of Amphs and their level changes upon the stimulation of cells were carried out with immunofluorescence microscopy. Confocal immunofluorescence microscopy was performed in VSMC by incubating cells with a mixture of clone 8-2 and 14-23. It was shown that the levels of Amphs increased slightly after treatment with insulin (Figure 5). These results suggest that our mAbs (clone 8-2 and 14-23) should be useful to study the cellular localization of Amphs.

\section{Discussion}

Amph1 is known as the autoantigen of Stiff-Man syndrome with breast cancer (De Camilli P. et al., 1993). The expression and purification systems of three separate domains of Amph1 established here should be useful in detecting antibodies of Stiff-Man syndrome. Attempt was made to solve difficulty of expressing whole large Amph1 molecule by subdividing the entire length by three domains hoping that there exist interdependent domains to maintain native conformation (structure-dependent epitopes). Purified Amph1N contains $50 \%$ of Amph1 from $\mathrm{N}$-terminus and purified 
A

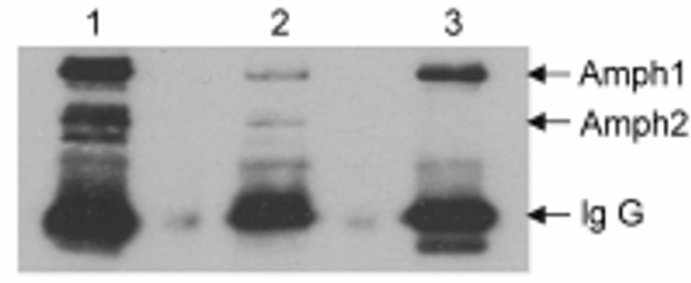

B

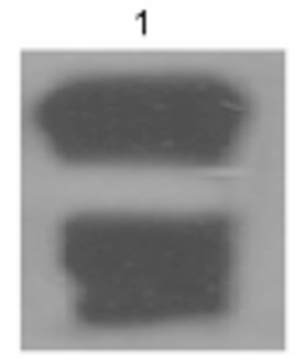

$\mathrm{C}$
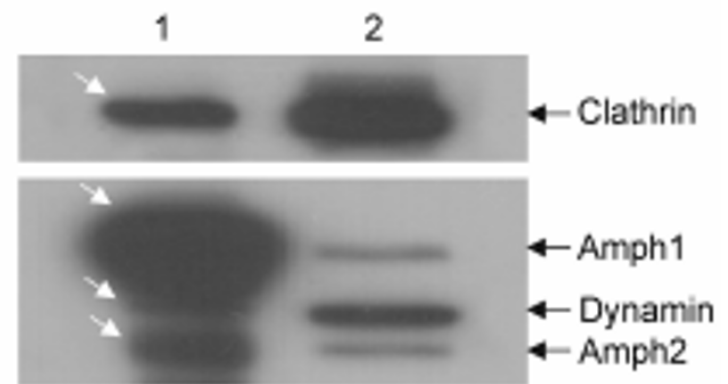

Figure 4. Immunoprecipitation analysis with monoclonal anti-amphiphysins. A, The lysates of several cell lines were centrifuged at $20,000 \mathrm{~g}$ for 1 h. The supernatants were precleared with protein A-gel and then subjected to immunoprecipitation with monoclonal anti-amphiphysins of three clones and protein A-gel. Immune complexes were collected and washed by a brief centrifugation, separated by SDS-PAGE on an $8 \%$ gel. Western blot analysis was done with monoclonal anti-amphiphysin. Lane 1, clone 14-23; lane 2, clone 14-19; lane 3, clone 8-2. B, After immunoprecipitation with clone 1423, Western blot analysis was performed with anti-amphiphysins (lane 1) and commercial anti-amphiphysin2 (lane 2). C, After immunoprecipitation with clone 8-2, precipitates were analyzed by repeated immunoblotting with anti-clathrin, antidynamin, and anti-amphiphysins as indicated. Lane 1, precipitate; lane 2, supernatant fraction.

Amph1C contains $60 \%$ of Amph1 from C-terminus. It means there is small (10\%) overlapping part. Because of some overlaps clone 8-2 recognizes both Amph1N and Amph1C. It can be concluded that clone 8-2 recognizes the middle part of Amph1.

Amphs have been reported to be specifically located in the nerve terminals and its mRNA is expressed in the brain and adrenal gland, but not in the liver, pancreas, spleen, testis or other tissues of chickens (Lichte et al., 1992). Our results were somewhat different. It is possible that the level of mRNA is inconsistent with the expression of protein. In our data, Amphs are expressed in the brain, lungs, and the testis of rats, while they are located only in the brain and the lungs of mice. Amph1 is present only in the cytosolic fraction in the lungs, whereas it is present both in the cytosolic and the membrane fractions of the brain (Figure $3 \mathrm{C}$ ). To understand the differential expression and cellular location of Amphs between species and organs a further study is required. Even though there are several isoforms of Amphs in PC12 cell, only a small band was present in the adrenal gland (Figure 3E). Although PC12 cells originated from the adrenal phenochromocytes, several isoforms of Amphs are highly expressed in PC12 cells but not in the adrenal gland. Whether Amphs are associated with cancer is not yet clear. It might be possible that Amphs are involved in the transformation of pheochromocyte.

Binding proteins with Amphs, i.e. dynamin and synaptojanin, have several isotypes (Ringstad et al., 1997). Dynamin I is a brain-specific isoform, dynamin II is expressed in all tissues, and dynamin III is most abundant in testis but also expressed in the brain and the lungs (Urrutia et al., 1997). Recently, the isotypes of Amph have been reported (Sakamuro et al., 1996; Butler, M.H et al., 1997; Kadlec et al., 1997; Leprince et al., 1997). Amph2 also binds to dynamin and synaptojanin through its $\mathrm{SH} 3$ domain. In vivo, Amph2 and dynamin can coimmunoprecipitate in the PC12 cells stimulated with NGF. It means that Amph2 may be connected to receptor-dependent signaling pathways (Leprince et al., 1997). The other isotype, BIN1 is a nuclear polypeptide. Distinctly, BIN1 is located in nucleus. It interacts with the $\mathrm{N}$-terminal region of $\mathrm{Myc}$ and inhibits malignant cell transformation by Myc. Besides Amph2, BIN1, and ALP1 (the Amph-like protein 1) interact functionally with the $\mathrm{CABL}$ tyrosin kinase $\mathrm{C}$-terminal via its $\mathrm{SH} 3$ domain in vitro and in vivo. Different from Amph1, ALP1 is highly expressed in skeletal muscles (Kadlec et al., 1997). Our anti-amphiphysin recognize two other proteins with molecular weights of $70 \mathrm{kD}$ and $90 \mathrm{kD}$ as well as Amph1. Those proteins were highly expressed in the brain and PC12 cells. In the $\mathrm{N}$-terminal anti-sense experiment, the $70 \mathrm{kD}, 90 \mathrm{kD}$ and Amph1 also changed to negative (data not shown). According to that data, It is believed that the $70 \mathrm{kD}, 90 \mathrm{kD}$ proteins may be other isoforms of Amphs.

To discover positive clones to precipitate Amphs, an immunoprecitation was performed. The results of immunoprecipitation were different from those of Western blotting. Though clone 14-23, 14-19, and 8-2 recognize several strong bands in Western blotting, the result of immunoprecipitation was different. Clone 14-23 also precipitates several isoforms of Amph, whereas clone 14-19 precipitates Amphs poorly, and clone 8-2 sediments only Amph1 in cell lysate (Figure 4A). To study the binding proteins with Amphs, it was tested whether antibodies block the interaction of Amphs and other proteins such as dynamin and clathrin. After immunoprecipitation, dynamin and clathrin are shown in Western blotting analysis (Figure 4C). 

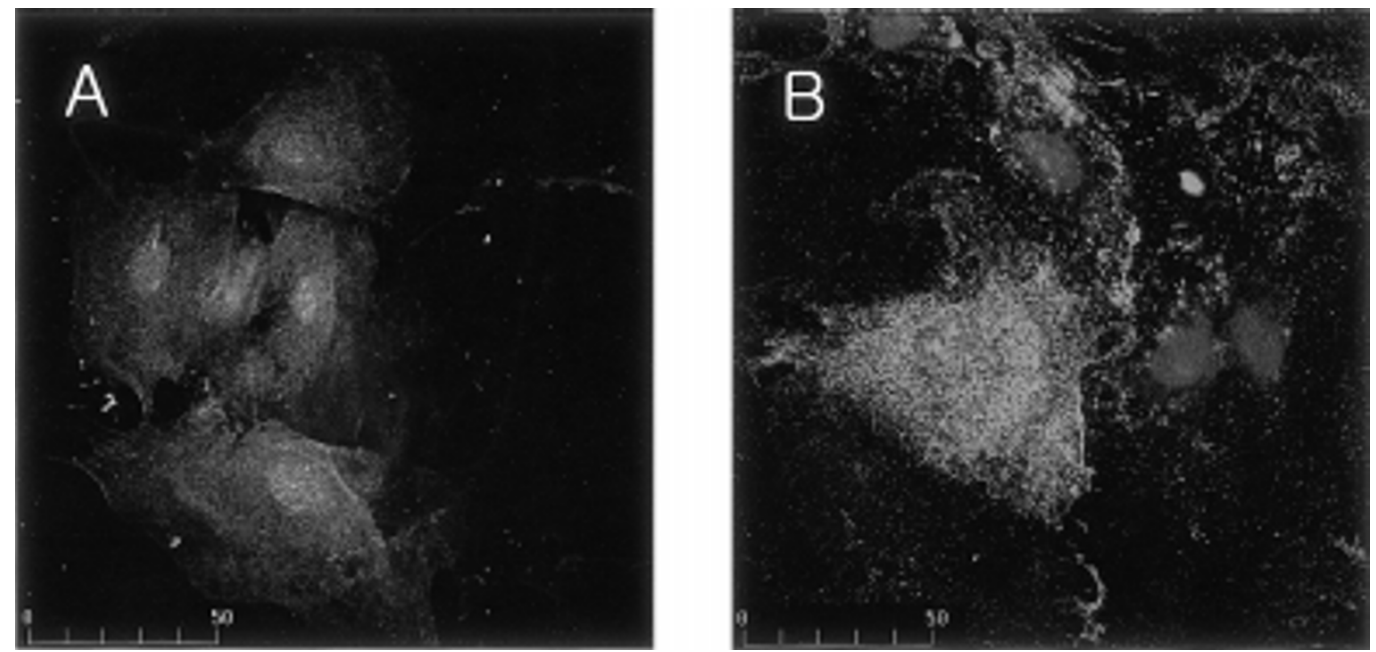

Figure 5. Confocal immunofluorescence microscopy with monoclonal antibodies against Amphs. Starved VSMC were incubated for 5 min in the absence (A) or presence(B) of insulin. Amphs were visualized using a mouse monoclonal anti-Amphs mixture (clones 4-2 and 8-2) and an FITC conjugated anti-mouse IgG. Nucleus was stained with propidium iodide $(\mathrm{Pl}, 10 \mu \mathrm{g} / \mathrm{ml})$ after cytosolic RNA was removed by RNase $\mathrm{l}-\mathrm{A}(0.5 \mathrm{mg} / \mathrm{ml})$.

To examine whether our mAbs can be used in immunocytochemistry. Confocal immunofluorescence microscopy was performed in VSMC. We were able to obtain good pictures. Taken together, these results suggest that our mAbs should be an excellent tool in studying the physiological roles of Amphs.

\section{Acknowledgements}

This work was supported by KOSEF (981-0704-029-2) and the grants of Post-Doc. Program (KOSEF, 1999).

\section{References}

Bauerfeind R, Takei K, De Camilli P. Amphiphysin I is associated with coated endocytic intermediates and undergoes stimulation-dependent dephosphorylation in nerve terminals. J Biol Chem 1997;272:30984-92

Bradford MM. A rapid and sensitive method for the quantitation of microgram quantities of protein utilizing the principle of protein-dye binding. Anal Biochem 1976;72:24854

Butler MH, David C, Ochoa GC, Freyberg Z, Daniell L, Grabs D, Cremona O, De Camilli P. Amphiphysin II (SH3P9; BIN1), a member of the amphiphysin/Rvs family, is concentrated in the cortical cytomatrix of axon initial segments and nodes of Ranvier in brain and around $\mathrm{T}$ tubules in skeletal muscle. $\mathrm{J}$ Cell Biol 1997;137:1355-67

De Camilli P, Thomas A, Cofiell R, Folli F, Lichte B, Piccolo G, Meinck HM, Austoni M, Fassetta G, Bottazzo G. The synaptic vesicle-associated protein amphiphysin is the 128-kD autoantigen of Stiff-Man syndrome with breast cancer. J Exp Med 1993;178:2219-23
Floyd SR, Porro EB, Slepnev VI, Ochoa GC, Tsai LH, De Camilli $P$. Amphiphysin binds the cdk5 regulatory subunit p35 and is phosphorylated by cdk5 and cdc2. J Biol Chem 2001; 276:8104-10

Kadlec L, Pendergast AM. The amphiphysin-like protein 1 (ALP1) interacts functionally with the $\mathrm{CABL}$ tyrosine kinase and may play a role in cytoskeletal regulation. Proc Natl Acad Sci U S A. 1997;94:12390-95

Lee C, Kim SR, Chung JK, Frohman MA, Kilimann MW, Rhee SG. Inhibition of phospholipase D by amphiphysins. J Biol Chem 2000;275:18751-58

Lee J-H, Kim SR, Chung HK. An enzyme immunoassay for pregnancy-specific- $\beta 1$ glycoprotein (SP1) employing monoclonal antibodies. Exp Mol Med 1996;28:181-86

Leprince C, Romero F, Cussac D, Vayssiere B, Berger R, Tavitian A, Camonis JH. A new member of the amphiphysin family connecting endocytosis and signal transduction pathways. J Biol Chem 1997;272:15101-5

Lichte B, Veh RW, Meyer HE, Kilimann MW. Amphiphysin, a novel protein associated with synaptic vesicles. EMBO J 1992;11:2521-30

Peters D, Tsuchida M, Manthei ER, Alam T, Cho CS, Knechtle SJ, Hamawy MM. Potentiation of CD3-induced expression of the linker for activation of $T$ cells (LAT) by the calcineurin inhibitors cyclosporin A and FK506. Blood 2000;95:2733-41

Ramjaun AR, McPherson PS. Multiple amphiphysin II splice variants display differential clathrin binding: identification of two distinct clathrin-binding sites. J Neurochem 1998;70: 2369-76

Ringstad N, Nemoto Y, De Camilli P. The SH3p4/Sh3p8/ SH3p13 protein family: binding partners for synaptojanin and dynamin via a Grb2-like Src homology 3 domain. Proc Natl Acad Sci U S A. 1997;5;94:8569-74

Sakamuro D, Elliott KJ, Wechsler-Reya R, Prendergast GC. 
BIN1 is a novel MYC-interacting protein with features of a tumour suppressor. Nat Genet 1996;14:69-77

Slepnev VI, Ochoa GC, Butler MH, Grabs D, Camilli PD. Role of phosphorylation in regulation of the assembly of endocytic coat complexes. Science 1998;281:821-24

Urrutia R, Henley JR, Cook T, McNiven MA. The dynamins: redundant or distinct functions for an expanding family of related GTPases. Proc Natl Acad Sci U S A 1997;94:377-84.
Review

Wigge P, Kohler K, Vallis $\mathrm{Y}$, Doyle CA, Owen D, Hunt SP, McMahon HT. Amphiphysin heterodimers: potential role in clathrin-mediated endocytosis. Mol Biol Cell 1997;8:2003-15

Wigge $\mathrm{P}$, McMahon HT. The amphiphysin family of proteins and their role in endocytosis at the synapse. Trends Neurosci 1998;21:339-44 